\title{
Combined approach for embolization of otherwise unmanageable gastric varices
}

\section{Anna Maria lerardia, Aldo Carnevale ${ }^{b}$, Giulia Tosettic, Mario D’Amicod, Melchiore Gigantie, Vincenzo La Muraf, Cristian Bonelliक, Matteo Renzullih, Pietro Lamperticoci, Massimo Primignanic, Gianpaolo Carrafielloa,j}

Fondazione IRCCS Cà Granda Ospedale Maggiore Policlinico, Milan; University Radiology Unit, Sant’Anna University Hospital, Ferrara; CRC "A. M. and A. Migliavacca" Center for Liver Disease, Milan; UOC Radiodiagnostica, Ospedali Riuniti Villa Sofia-Cervello, Palermo; University of Ferrara; University of Milan; Foundation IRCCS Cà Granda Ospedale Maggiore Policlinico, Milan; Sant'Orsola Hospital, University of Bologna; University of Milan, Milano, Lombardia; Università degli Studi di Milano, Milan, Italy

\section{Abstract}

\begin{abstract}
Background This study aimed to determine the feasibility, safety and effectiveness of combined percutaneous transhepatic obliteration (PTO) and balloon-occluded retrograde transvenous obliteration (BRTO) therapy for the treatment of patients with high-risk bleeding gastric varices.
\end{abstract}

\begin{abstract}
Methods Ten patients were retrospectively reviewed. All the patients presented gastric varices, according to the Sarin classification, at high risk of bleeding, and not otherwise manageable. Patients with portal vein thrombosis were excluded. All patients were treated with a combination of PTO and BRTO. In all cases the gastric varices were embolized with glue, combined with coils or not, with an occlusion balloon inflated into the shunt. In 7 cases, embolization was immediate; in the remaining 3 the balloon remained inflated for $4 \mathrm{~h}$ and in 2 of them embolization of the shunt was required. Technical success was defined as complete obliteration of the gastric varices observed during a contrast-enhanced computed tomography study and endoscopy within 1 month following treatment. Clinical success was defined as absence of bleeding of gastric varices during the follow-up period. Major and minor complications during the follow up were recorded.
\end{abstract}

Results Twelve sessions of combined PTO and BRTO procedures were performed in 10 patients; in 2 patients a new combined treatment was required during the follow up. Technical and clinical success was $100 \%$. Neither major nor minor procedure-related complications were observed.

Conclusion Combined PTO and BRTO therapy is safe and effective for the treatment of gastric varices that cannot be managed otherwise.

Keywords Gastrointestinal hemorrhage, portal hypertension, gastric varices, balloon-occluded retrograde transvenous obliteration, percutaneous transhepatic obliteration

Ann Gastroenterol 2021; 34 (2): 1-6

\section{Introduction}

Varices due to portal hypertension are abnormally dilated submucosal veins that can occur throughout the digestive

\section{Conflict of Interest: None}

Correspondence to: Anna Maria Ierardi, MD, Radiology Department, Fondazione IRCCS Cà Granda Ospedale Maggiore Policlinico, Via Francesco Sforza, 35, 20122, Milan, Italy, e-mail: amierardi@yahoo.it

Received 26 November 2020; accepted 28 January 2021; published online 23 March 2021

DOI: https://doi.org/10.20524/aog.2021.0616 tract and can lead to life-threatening bleeding. Variceal hemorrhage is definitely a medical emergency, associated with a mortality that is still around $10-20 \%$ at 6 weeks, despite recent developments and refinement of technologies and treatment approaches [1,2]. Optimal management requires a thoughtful, multidisciplinary strategy, including gastroenterologists or hepatologists, and diagnostic and interventional radiologists.

Esophagogastroduodenoscopy is traditionally considered the first-line diagnostic and therapeutic tool for bleeding esophageal and gastric varices [1,3]. Without endoscopic intervention, the risk of rebleeding is almost $60 \%$, with a higher mortality rate (33\%) [4-6]. Rebleeding may be 
managed by a second attempt at endoscopy; however, in the case of failure of endoscopic treatment and severe hemorrhage, a transjugular intrahepatic portosystemic shunt (TIPS) is likely to be the best strategy [1], in line with the traditional doctrine of decompressing the hypertensive portal circulation.

However, TIPS has not proved to be as effective in treating gastric variceal hemorrhage as it has in the case of esophageal variceal bleeding [7]. Indeed, in most cases gastric varices are supplied by a spontaneous splenorenal shunt, and they bleed with a lower portosystemic gradient than esophageal varices. In this scenario, balloon-occluded retrograde transvenous obliteration (BRTO) has been gaining popularity in the scientific literature, especially in Asian countries [6,7].

The traditional technique consists of retrograde catheterization of the left renal vein by the jugular or femoral vein, followed by occlusion through a balloon and slow infusion of glue to achieve obliteration of the gastro-splenorenal collateral pathways and gastric fundal varices. This procedure, with variations and possibly combinations of techniques, has not only proved to be effective in controlling gastric variceal bleeding, with low recurrence rates, but also reveals some advantages over TIPS, particularly in terms of hepatic encephalopathy and liver insufficiency. The effect of the former procedure is the obliteration of a spontaneous hepatofugal shunt, this being the opposite of the aforementioned principle of portal decompression. However, BRTO may be challenging or not suitable in certain settings, as in the case of varices that lack a main draining vein, or in the presence of portosystemic shunts with complex anatomy. In these circumstances, a combination of percutaneous transhepatic obliteration (PTO) with BRTO, despite its greater invasiveness, may be the best strategy [8].

In this article, we sought to determine the feasibility, safety and effectiveness of combined PTO and BRTO therapy for the treatment of patients with high-risk bleeding gastric varices.

${ }^{a}$ Radiology Department, Fondazione IRCCS Cà Granda Ospedale Maggiore Policlinico, Milan (Anna Maria Ierardi, Gianpaolo Carrafiello); ${ }^{\circ}$ Radiology Department, University Radiology Unit, Sant'Anna University Hospital, Ferrara (Aldo Carnevale); 'Foundation IRCCS Ca' Granda Ospedale Maggiore Policlinico, Division of Gastroenterology and Hepatology, CRC "A. M. and A. Migliavacca" Center for Liver Disease, Milan (Giulia Tosetti, Massimo Primignani); ${ }^{\mathrm{d} U O C}$ Radiodiagnostica; Ospedali Riuniti Villa Sofia-Cervello, Palermo (Mario D’Amico); ${ }^{\text {eDepartment of }}$ Morphology, Surgery and Experimental Medicine, University of Ferrara (Melchiore Giganti); ${ }^{\mathrm{f}}$ Fondazione IRCCS Ca' Granda, Ospedale Maggiore Policlinico, U.O.C. Medicina Generale Emostasi e Trombosi, University of Milan (Vincenzo La Mura); ${ }^{8}$ Healthcare Professionals Department, Foundation IRCCS Cà Granda Ospedale

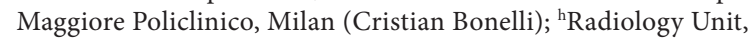
Department of Experimental, Diagnostic and Speciality Medicine, Sant'Orsola Hospital, University of Bologna (Matteo Renzulli); ${ }^{i}$ Department of Pathophysiology and Transplantation, University of Milan, Milano, Lombardia (Pietro Lampertico); ${ }^{j}$ Department of Health Sciences, Università degli Studi di Milano, Milan (Gianpaolo Carrafiello), Italy

\section{Patients and methods}

\section{Patients}

From January 2017 to August 2020, 10 patients (9 men and 1 woman; median age 67.5 years) were retrospectively reviewed (Table 1). All the patients had been treated with a combination of PTO and BRTO. Inclusion criteria were: liver cirrhosis with high-risk bleeding gastric varices; uncontrolled hepatic encephalopathy; uncontrolled gastric varices despite previous endoscopic treatment; contrast-enhanced computed tomography (CECT); and/or endoscopy performed before the procedure for evaluation of gastric and esophageal varices. Patients with portal vein thrombosis or severe uncorrected coagulopathy were excluded. Gastric fundal varices were classified according to the classification system originally proposed by Sarin et al [9]. Written informed consent was acquired from all the patients enrolled prior to treatment.

The series was a retrospective cohort study, conducted in accordance with the Declaration of Helsinki.

\section{Procedure}

All procedures were performed in the angiographic suite (GE-Innova 2100-IQ, GE Healthcare, USA) by two interventional radiologists with more than 10-year experience in endovascular and percutaneous techniques, with the patient under conscious sedation and local anesthesia at the puncture site using lidocaine hydrochloride. All patients received antibiotic prophylaxis according to an "ultra-shortterm antibiotic prophylaxis" scheme with a preprocedural intravenous injection of $2 \mathrm{~g}$ of cefazolin.

As planned before the intervention, all patients were treated with a combined approach: percutaneous access was obtained under fluoroscopic guidance using a 21-G needle (AccuStick System, Boston Scientific) and a 4-5-Fr sheath. In 2 patients, paracentesis was needed before the procedure (within $24 \mathrm{~h}$ ) to permit a safe percutaneous liver puncture. Retrograde catheterization of splenorenal shunts via transvenous femoral or jugular puncture was also performed. In all cases, gastric varices were visualized by injection of contrast media from the splenic side; embolization was performed from this side after a super-selective catheterization of the varices. An occlusion balloon was inflated into the shunt from the renal side to avoid the migration of the embolic agents into the systemic circulation (Fig. 1A,B). Embolization was performed with glue in all cases

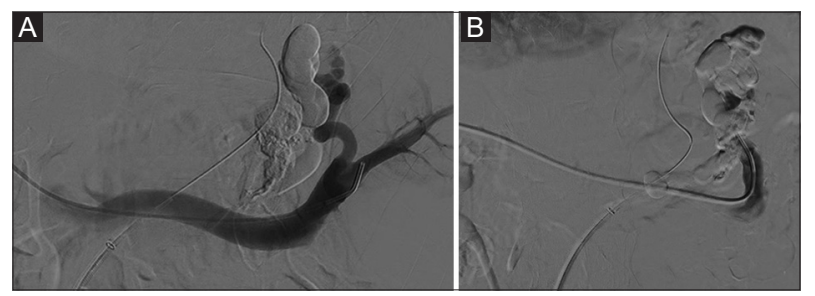

Figure 1 Intra-procedural images during embolization with glue (A) and at the end of the procedure (B) 
Combined BRTO and PTO therapy for gastric varices 3

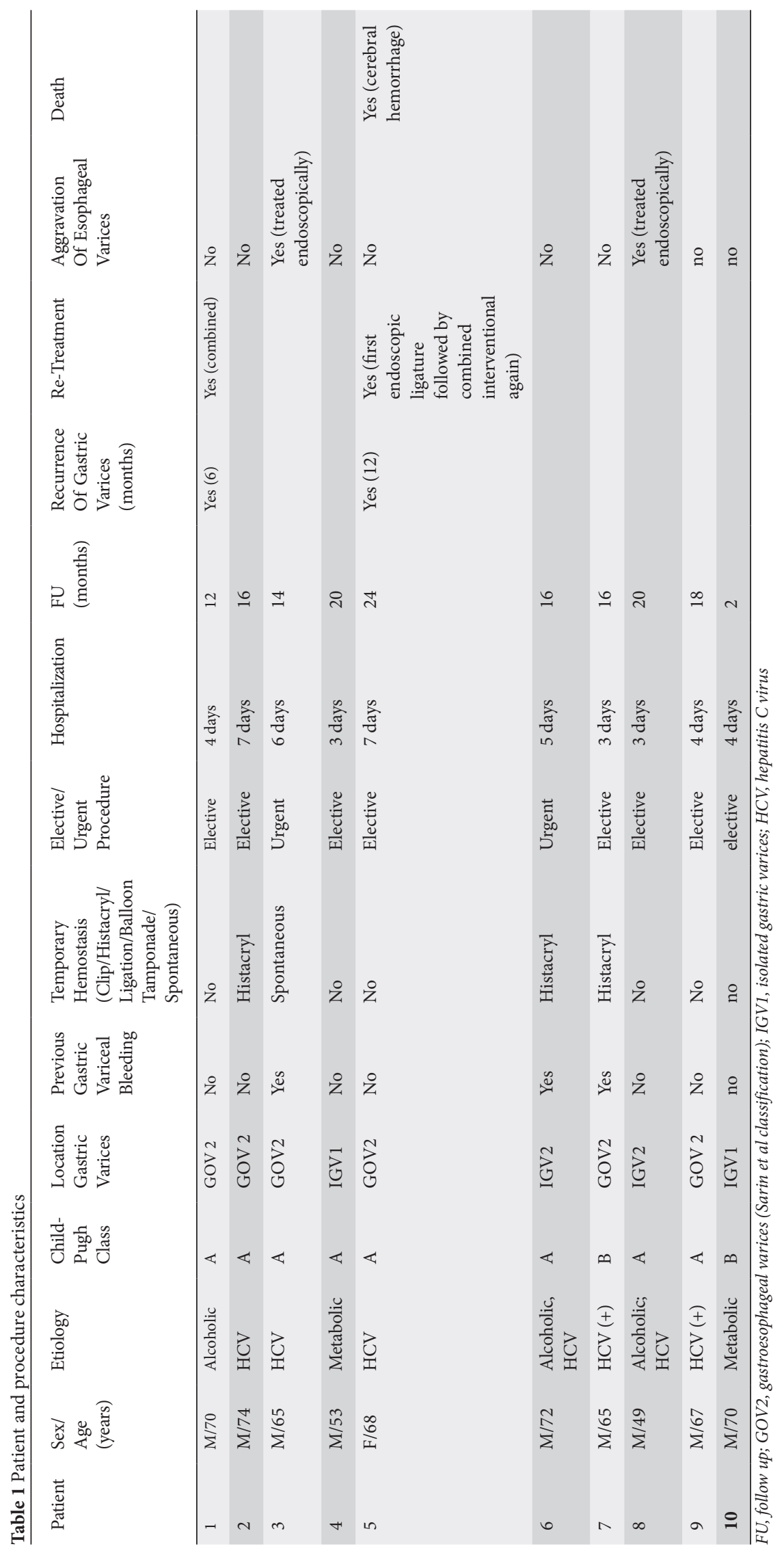


except for one, in which coils were coated with cyanoacrylate: in this way, the coils served as a scaffold to which the glue could anchor, in order to reduce the risk of glue migration. Glue was used in different combinations with lipiodol, depending on the polymerization time desired: we started by using a rate of 1:2 and then continued with 1:1. In all cases, glue was delivered via a 2.7-Fr microcatheter (Progreat, Terumo, Japan), and 5-Fr diagnostic catheters, Cobra $\mathrm{C} 1$ or vertebral or ber (Cordis, Miami Lakes, FL, USA) were used to catheterize the shunts.

In 7 cases, embolization was immediate and the occlusion balloon was deflated at the end of the procedure; in 3 cases the balloon was left inflated for $4 \mathrm{~h}$ to facilitate hemostasis, and in 2 of these patients an additional occlusion of the shunt was required and performed with plug.

The percutaneous liver approach was closed with a 4-mm AMPLATZER vascular plug (AVP)-IV (AVP; St. Jude Medical, St. Paul, MN) in 5 cases and with a 5-mm AVP-IV in 1 case; no embolization of the percutaneous approach was performed in the remaining cases.

Complications were graded as major or minor, following the Society of Interventional Radiology's classification system [10].

\section{Follow up}

Immediately after the interventional procedure, varices were assessed by direct portography. Technical success was defined as complete obliteration of the gastric varices observed during CECT study and endoscopy within 1 month from the treatment (Fig. 2A-D and Fig. 3A,B). Thereafter, endoscopy was performed every 3 months.

Clinical success was defined as the absence of gastric varices at high risk of bleeding during the available follow-up period. The follow-up period for the recurrence of high-risk gastric varices was calculated from the date of the procedure until endoscopy or CT showed morphologic recurrence. For endoscopy that meant the reappearance of swelling gastric varices with signs of high risk of hemorrhage (hyperemia of the mucosa, initial bleeding). For CT, a reappearance of vascularized fundal gastric varices was considered as recurrence. A new combined treatment was considered if endoscopy was not successful.

Follow-up evaluation included the assessment of recurrence of high-risk gastric varices, complications, and survival rate. In addition, hepatic function tests, including serum albumin, bilirubin, transaminases, ammonia concentrations, and prothrombin time at discharge and 15 days after gastric varices obliteration, were performed during the follow-up period. The survival period was assessed from the date of the procedure until death or the most recent clinical visit.

\section{Results}

Patient characteristics and procedural data are summarized in Table 1. Twelve sessions of combined PTO and BRTO

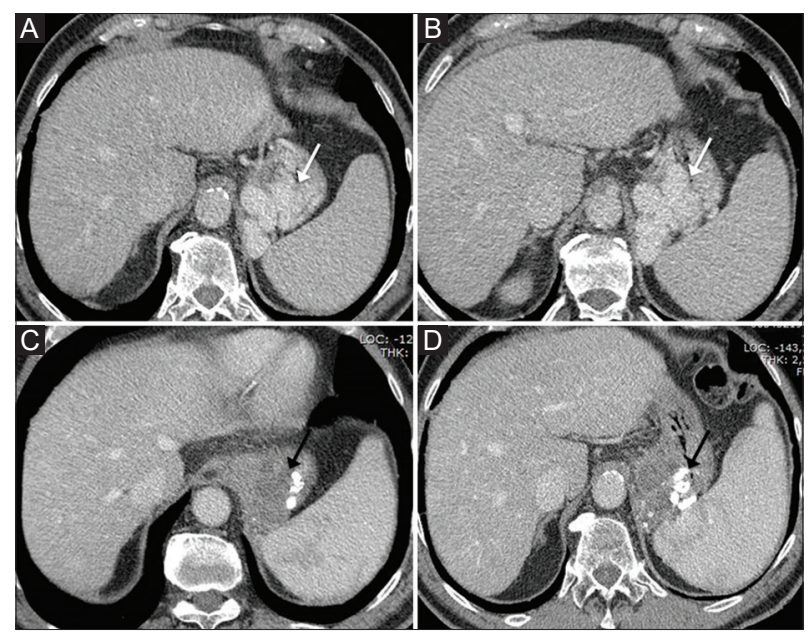

Figure 2 Contrast-enhanced computed tomography (CT) scan demonstrates enhancing dilated gastric varices extending to the gastric mucosal surface (white arrows) (A, B). Contrast-enhanced axial CT images obtained after embolization show unenhanced fundal gastric varices with embolic material inside (black arrows) (C, D)
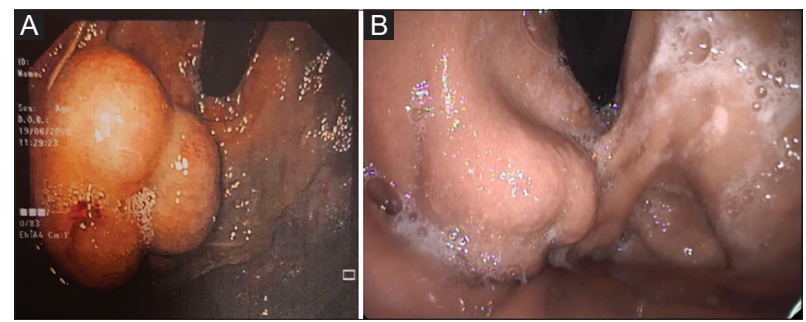

Figure 3 Endoscopic image of the gastric varices, before the treatment (A) and at 1-month follow up after successful treatment (B)

procedures were performed in 10 patients. The mean hospital stay after treatment was $4.6 \pm 1.5$ days. Technical and clinical success was $100 \%$. The mean follow-up duration was $15.8 \pm 5.6$ months. During the observation period, gastric varices recurred in 2 patients and a further combined treatment was successfully performed. In one of these, endoscopic ligature was attempted first, but it was not effective.

Neither major nor minor procedure-related complications occurred, except for mild abdominal pain in 2 cases, which resolved in $24 \mathrm{~h}$ without any treatment. Esophageal varices worsened in 2 of 10 patients who underwent endoscopic band ligation. One patient died 12 months after the combined treatment because of cerebral hemorrhage.

\section{Discussion}

Variceal hemorrhage is a fearful complication of portal hypertension, and represents a leading cause of mortality in patients with cirrhosis [11]. Although gastric varices carry a lower risk of bleeding than esophageal varices, they tend to bleed profusely once ruptured, with a higher mortality rate $[8,12]$. Several therapeutic modalities are 
currently available to treat high-risk gastric varices, including endoscopic procedures, TIPS, PTO and BRTO. However, the best approach for controlling variceal gastric hemorrhage remains to be established [8]. Shunting procedures, either surgical or endovascular, are effective in decompressing portal hypertension, but may lead to severe complications, mainly hepatic encephalopathy and liver failure. Moreover, TIPS has not proved to be as effective in controlling gastric variceal hemorrhage as it has with esophageal variceal bleeding [7], and high-risk varices, such as those in the gastric antrum, may still be at risk of bleeding even after the portosystemic gradient has been reduced [13].

The available literature suggests that BRTO therapy and its variations may offer an effective alternative to TIPS in the case of active bleeding, uncontrolled by medical or endoscopic management, or to prevent re-bleeding, especially in patients who have appropriate shunts and may otherwise not tolerate TIPS (e.g., those with refractory hepatic encephalopathy or elderly subjects) [14-16]. Moreover, BRTO carries the potential advantage over TIPS of increasing portal blood flow and potentially improving liver function.

Broadly speaking, transvenous obliteration of gastric varices can be achieved from the systemic venous side (draining veins/shunts) or from the portal venous side (portal afferent feeding vessels). Through the combined approach we have described, the varices in our series were approached simultaneously from the systemic side by BRTO and from the portal side by PTO.

BRTO is an increasingly accepted, minimally invasive treatment for high-risk gastric varices, carrying a high technical success rate and a low rate of major complications $[8,11,15,17-22]$. The main clinical indications for BRTO are gastric variceal bleeding (impending, prior or active) and, to a lesser extent, refractory severe encephalopathy [23]. A metanalysis [12] has shown a pooled clinical success rate of $97.3 \%$ with a low incidence of major complications. Common side effects reported are minor and temporary, including hematuria, ascites and fever. After the first description of the technique by Kanagawa et al [24], additional options have been provided to force retrogradely sclerosant agents into the varices, while occluding the outflow via a balloon. Indeed, since in many cases the anatomy of gastric varices is complex because of multiple feeding and draining routes [13], combining anterograde and retrograde approaches is frequently useful. Alternative routes for transvenous obliteration are often sought, in addition to the classical transrenal access, since the procedure carries a potential for many adjuvant techniques and complimentary approaches $[17,25]$.

Unfortunately, the BRTO approach is sometimes not suitable or may be challenging-e.g., if there is no main draining vein, or in the presence of complex vascular connections. In these cases, PTO, alone or in combination with BRTO, may represent the best approach, able to achieve a better control of obliteration of collateral pathways by obstructing both the feeding and the draining veins of the varices [17]. PTO, first devised by Lunderquist and Vang in 1974, has been widely performed as an emergency measure in cases of variceal hemorrhage, aiming to obtain bleeding control through antegrade embolization via transhepatic puncture [19].

In general, PTO is considered an adjunct or an alternative to BRTO, when the latter fails to obliterate the gastric variceal system, or is technically challenging and requires additional portal access [26]. Bailout antegrade transvenous obliteration of initially failed or challenged BRTO has been proven to increase the technical and hemodynamic success rate of transvenous obliteration of gastric varices from 84-98\% (BRTO only approach) to $98-100 \%$ (combined approach) [26].

In our series, the combination of the 2 techniques, although obviously more invasive than BRTO alone, was effective and safe in all cases; notably, the percutaneous approach allowed us to check for and carefully avoid the retrograde migration of the embolic agent into the portal vein and/or splenic vein. In our opinion, as previously reported [27], a combined strategy, which can obliterate both the feeding and the draining veins with the aim of forcing the sclerosing agent completely into the varices, may yield better control of the variceal blood flow than that offered by BRTO or PTO therapy alone. By this method, the varix is indeed "trapped" simultaneously from the portal venous side (PTO) and from the transrenal/systemic venous side (BRTO) with the occlusion balloons. This enables adequate sclerosant distribution.

Unlike TIPS, BRTO does not divert portal blood flow from the liver, while it increases the hepatopetal blood flow by obliterating the hepatofugal shunts [15]. Therefore, though aggravating portal hypertension, it does not deteriorate liver function and could be preferable in patients with very advanced cirrhosis or hepatic encephalopathy. Similar changes in hemodynamics, also expected with combined PTO and BRTO [8], may exacerbate the sequelae of portal hypertension, with increased incidence or worsening of preexisting esophageal varices and ascites $[11,12]$. Thus, early endoscopic follow up is necessary after such interventional treatments of gastric varices, particularly in patients with preexisting esophageal varices, and esophageal varices at bleeding risk should be treated with endoscopic band ligation. Though such possible adverse events are disappointing, one should consider that the mortality rate for gastric variceal hemorrhage is higher than that for esophageal variceal bleeding and, in accordance with Arai et al, should conclude that the expected changes in portal system hemodynamics caused by the shunt obliteration are still preferable, since esophageal varices can be controlled more easily by endoscopic thrapy [27].

Our work has several limitations, including the retrospective study design and the limited number of patients; further prospective, randomized, comparative studies with larger cohorts and a longer follow-up period would assist in clarifying the effect of combined BRTO and PTO treatment.

In conclusion, our preliminary experience shows that combined PTO and BRTO therapy is feasible, safe and effective in treating uncontrolled gastric varices. This strategy offers an attractive alternative for patients with high-risk bleeding gastric varices that cannot be managed otherwise, providing optimal control of the variceal blood flow. 


\section{Summary Box}

\section{What is already known:}

- Although gastric varices carry a lower risk of bleeding than esophageal varices, they tend to bleed profusely once ruptured and have a higher mortality rate

- The balloon-occluded retrograde transvenous obliteration (BRTO) procedure, with variations and possibly combinations of techniques, has proved to be effective in controlling gastric variceal hemorrhage

\section{What the new findings are:}

- Combined percutaneous transhepatic obliteration (PTO) and BRTO therapy is safe and effective

- It may rescue cases with otherwise uncontrollable gastric varices

- A combined PTO and BRTO approach may provide optimal control of the variceal blood flow, avoiding migration of the embolic agents

\section{References}

1. Kamath PS, Mookerjee RP. Individualized care for portal hypertension: Not quite yet. J Hepatol 2015;63:543-545.

2. Ierardi AM, Piacentino F, Pesapane F, et al. Basic embolization techniques: tips and tricks. Acta Biomed 2020;91:71-80.

3. Ierardi AM, Del Giudice C, Coppola A, et al. Gastrointestinal hemorrhages in patients with covid-19 managed with transarterial embolization. Am J Gastroenterol 2020 Oct 1 [Online ahead of print] doi: 10.14309/ajg.0000000000000978

4. Boregowda U, Umapathy $\mathrm{C}$, Halim $\mathrm{N}$, et al. Update on the management of gastrointestinal varices. World J Gastrointest Pharmacol Ther 2019;10:1-21.

5. Hernández-Gea V, Procopet B, Giráldez, et al. Preemptive-TIPS improves outcome in high-risk variceal bleeding: an observational study. Hepatology 2018;69:282-293.

6. Nicoară-Farcău O, Han G, Rudler $M$, et al. Effects of early placement of transjugular portosystemic shunts in patients with high-risk acute variceal bleeding: a meta-analysis of individual patient data. Gastroenterology 2020;160:193-205.e10.

7. Saad WE. Introduction: BRTO \& BATO. Tech Vasc Interv Radiol 2013; 16:59.

8. Uchiyama F, Murata S, Onozawa S, et al. Management of gastric varices unsuccessfully treated by balloon-occluded retrograde transvenous obliteration: long-term follow-up and outcomes. ScientificWorldJournal 2013;2013:498535.

9. Sarin SK, Lahoti D, Saxena SP, Murthy NS, Makwana UK. Prevalence, classification and natural history of gastric varices: a long-term follow-up study in 568 portal hypertension patients. Hepatology 1992;16:1343-1349.
10. Sacks D, McClenny TE, Cardella JF, Lewis CA. Society of Interventional Radiology clinical practice guidelines. J Vasc Interv Radiol 2003;14:S199-S202.

11. Lipnik AJ, Pandhi MB, Khabbaz RC, Gaba RC. Endovascular treatment for variceal hemorrhage: TIPS, BRTO, and combined approaches. Semin Intervent Radiol 2018;35:169-184.

12. Park JK, Saab S, Kee ST, et al. Balloon-occluded retrograde transvenous obliteration (BRTO) for treatment of gastric varices: review and meta-analysis. Dig Dis Sci 2015;60:1543-1553.

13. Saad WE. Vascular anatomy and the morphologic and hemodynamic classifications of gastric varices and spontaneous portosystemic shunts relevant to the BRTO procedure. Tech Vasc Interv Radiol 2013;16:60-100.

14. Tripathi D, Stanley AJ, Hayes PC, et al; Clinical Services and Standards Committee of the British Society of Gastroenterology. U.K. guidelines on the management of variceal haemorrhage in cirrhotic patients. Gut 2015;64:1680-1704.

15. Garcia-Tsao G, Abraldes JG, Berzigotti A, Bosch J. Portal hypertensive bleeding in cirrhosis: Risk stratification, diagnosis, and management: 2016 practice guidance by the American Association for the Study of Liver Diseases. Hepatology 2017;65:310-335.

16. Vine LJ, Subhani M, Acevedo JG. Update on management of gastric varices. World J Hepatol 2019;11:250-260.

17. Saad WE, Sze DY. Variations of balloon-occluded retrograde transvenous obliteration (BRTO): balloon-occluded antegrade transvenous obliteration (BATO) and alternative/adjunctive routes for BRTO. Semin Intervent Radiol 2011;28:314-324.

18. Chang MY, Kim MD, Kim T, et al. Plug-assisted retrograde transvenous obliteration for the treatment of gastric variceal hemorrhage. Korean J Radiol 2016;17:230-238.

19. Ishikawa T, Imai $M$, Ko $M$, et al. Percutaneous transhepatic obliteration and percutaneous transhepatic sclerotherapy for intractable hepatic encephalopathy and gastric varices improves the hepatic function reserve. Biomed Rep 2017;6:99-102.

20. Gwon DI, Ko GY, Kwon YB, Yoon HK, Sung KB. Plug-assisted retrograde transvenous obliteration for the treatment of gastric varices: the role of intra-procedural cone-beam computed tomography. Korean J Radiol 2018;19:223-229.

21. Kim T, Yang H, Lee CK, Kim GB. Vascular plug assisted retrograde transvenous obliteration (PARTO) for gastric varix bleeding patients in the emergent clinical setting. Yonsei Med J 2016;57:973-979.

22. Minamiguchi $\mathrm{H}$, Kawai N, Sato $M$, et al. Balloon-occluded retrograde transvenous obliteration for gastric varices via the intercostal vein. World J Radiol 2012;4:121-125.

23. Saad WE, Kitanosono T, Koizumi J, Hirota S. The conventional balloon-occluded retrograde transvenous obliteration procedure: indications, contraindications, and technical applications. Tech Vasc Interv Radiol 2013;16:101-151.

24. Kanagawa H, Mima S, Kouyama H, Gotoh K, Uchida T, Okuda K. Treatment of gastric fundal varices by balloon-occluded retrograde transvenous obliteration. J Gastroenterol Hepatol 1996;11:51-58.

25. Patel M, Molvar C. Evolution of retrograde transvenous obliteration techniques. Semin Intervent Radiol 2018;35:185-193.

26. Saad WE, Kitanosono T, Koizumi J. Balloon-occluded antegrade transvenous obliteration with or without balloon-occluded retrograde transvenous obliteration for the management of gastric varices: concept and technical applications. Tech Vasc Interv Radiol 2012; 15:203-225.

27. Arai H, Abe T, Takagi H, Mori M. Efficacy of balloon-occluded retrograde transvenous obliteration, percutaneous transhepatic obliteration and combined techniques for the management of gastric fundal varices. World J Gastroenterol 2006;12:3866-3873. 